\title{
How Dental Professional Are Combating Opioid Addiction
}

\author{
Omar Alkhadra and Joseph Krajekian* \\ Department of Oral \& Maxillofacial Surgery, USA
}

Submission: November 11, 2020; Published: November 30, 2020

*Corresponding author: Joseph Krajekian, Department of Oral \& Maxillofacial Surgery, Ohio, USA

\section{Keywords: Bupivacaine; Dentists; Dihydrocodeine; Gum mastic; Nociceptors}

Keywords: OTC: Over the Counter; VR: Virtual Reality; CDC: Centers for Disease Control; ADA: American Dental Association; NSAIDs: Non-

Steroidal Anti-Inflammatory Drugs; APAP: Acetyl-Para-Aminophenol; VGNaC: Voltage-Gated Sodium Channel

\section{Introduction}

To create a comprehensive picture of the opioid crisis, it is important to understand the reason behind the crisis, pain. The mechanism of pain is quite complex, none the less we cannot survive without it. Pain is a defense mechanism that helps us protect ourselves from harmful situations or inform us to pay attention to something that is going wrong in our bodies. For example, a person will know that a stove is hot before even touching it because the hand will sense the heat radiating from the stove as it gets closer. Pain can also present as an early warning sign of arthritis, an appendix infection, or even a cavity. The opioid crisis has plagued the world since the beginning of the $21^{\text {st }}$ century. It has affected the sick and the healthy, the poor and the rich, and the young and the elderly with no discrimination. You might likely know of a family member or a friend who suffers from opioid dependency. As dentists and physicians, this issue is particularly important to us, as we are the first barrier between the general public and an opioid prescription (Figure 1).

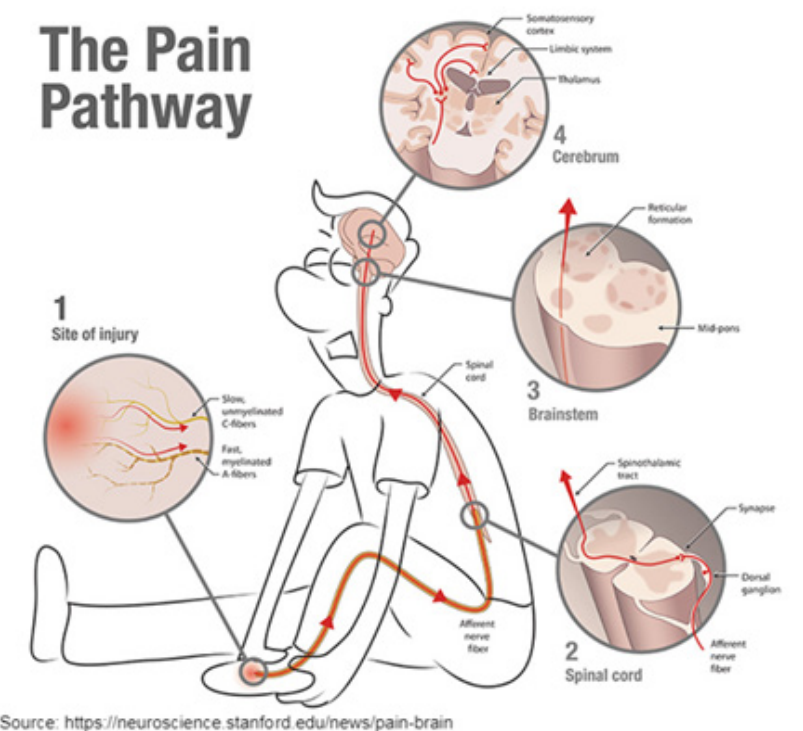

Figure 1: 


\section{Short Communication}

Understanding how pain works in the body will explain why this problem is so complex. The mechanism of pain is as follows; When tissue damage is initiated, special nerve cell endings called nociceptors send pain signals from the source of pain to the closest area of the spinal cord via the 1 st order neurons. The $1^{\text {st }}$ order neurons will then release a chemical called "substance P" into the synaptic cleft where a $2^{\text {nd }}$ order neuron will pick up the pain signal and transmit it to the thalamus in the brain via the spinothalamic tract. The thalamus is considered the relay station inside the brain. In the thalamus, a $3^{\text {rd }}$ order neuron will pick up the signal and relay it to the area of the brain which correlates to the source of the pain. Pain comes in two forms that are transmitted by two different fibers. The Alpha-Beta (A $\delta$ ) fibers that transmit a quick, sharp, and localized pain stimulus. And C fibers that transmit slow, dull, burning, or throbbing pain stimulus. The $\mathrm{A} \delta$ fibers allow the person to retract themselves away from harm even before pain is felt. This happens because these fibers are myelinated and allow signals to travel at a rate of $20 \mathrm{~m} / \mathrm{s}$ giving the body a chance to react even before the onset of pain is felt in the damaged area. On the other hand, $\mathrm{C}$ fibers are not myelinated and transmit signals at a much slower rate of about $0.5-2 \mathrm{~m} / \mathrm{s}$. The $\mathrm{C}$ fibers is what causes chronic or continuous pain. Long term pain management revolves around the $\mathrm{C}$ fibers as at the tips of the $\mathrm{C}$ fibers are the opioid receptors [1].

Controlling pain in the dental office has not always been as simple as a 30-second injection of local anesthesia Lidocaine that would allow for a smooth and seamless dental procedure. Pain was and still is the biggest factor in the dental field. It accounts for more than 80 percent of all dental visits [2,3]. Historically, humans tried many different methods to control pain. The earliest records available that indicate some of the methods used for dental pain management were found on a Babylonian clay tablet from 2250 B.C. It described a method of mixing henbane seed with gum mastic, the mixture is then inserted into the cavity of the aching tooth. Other methods include using alcohol on the area of surgery, or wine to get into a state of euphoria. Some evidence shows some cultures using opium saturated sponges placed on the nose, allowing the patient to breathe in the opium until they lose their senses. Also, pinching the carotid artery to induce unconsciousness was used by Egyptian and Assyrian physicians [4].

Since 2250 B.C., the dental field has advanced significantly in the management of pain. Dentists now have many tools in their arsenal to control pain during procedures like, nitrous oxide originally discovered by Humphry Davy [5], and local anesthesia that was first synthesized by Alfred Einhorn [6]. Tools for postprocedural pain management include the use of over the counter (OTC) and prescription medications. The excessive use of these prescription drugs is where the opioid crisis problem lies. There has been a lot of controversy about pharmaceutical companies overselling their drugs to physicians with false claims about safety and the rate of dependency. In fact, pharmaceutical companies had such a big role in the opioid crisis that as recently as August 2019, the health care giant Johnson and Johnson (J\&J) was ordered to pay more than 570 million dollars to the state of Oklahoma. The state argues that J\&J had falsely advertised the safety and underestimated the dangers of opioids. The state of Oklahoma has also sued Purdue Pharma and Teva Pharmaceuticals for their roles in the opioid crisis. Both companies have settled before going to trial for $\$ 270$ million and $\$ 85$ million dollars respectively [7]. Similarly, Purdue Pharma the manufacturer behind OxyContin aggressively marketed its product to doctors as a less addictive pain killer that can be used to treat common conditions like backaches and knee pain [8]. As a result, many doctors overprescribed opioid drugs to patients until patients unknowingly developed a dependency. Eventually, patients no longer have access to prescription pain killers and resolve to illegal ways of obtaining them. According to the National Institute on Drug Abuse article, the vast majority of individuals who misused pain killers obtained their drugs from a friend or relative after they run out [9].

Moreover, the culture of prescription drugs in the united states was very lenient prior to 2017 when compared to other developed countries. The United States represents approximately 5 percent of the world population yet consumes more than 80 percent of the worldwide opioid supply [10]. A comparison between American and English dentists shows that US dentists write prescriptions at a rate that is 37 times greater than their English counterparts [11]. The same study also shows that US dentists prescribe a wide array of prescription painkillers including hydrocodone, codeine, oxycodone, and tramadol. While English dentists used dihydrocodeine as the main and only prescription pain killer (Figure 2). A study conducted in 2019 compared the positive steps taken by several countries to mitigate opioid misuse. While it shows that the United States has higher usage per capita, it also explains the steps taken by governmental bodies to control the crises. The steps include targeting the number of opioids per patient, limiting the type of clinicians allowed to prescribe opioids, and increasing opioid substitution treatment programs [12].

After focusing on how pain works in our bodies, how opioids are integrated within our culture, and the infiltration of pharmaceutical companies into our health care system to increase their bottom line; what are the available alternatives to help patients become comfortable before, during and after dental procedures while keeping the risk of opioid dependency to a minimum. Given that dentists are on the front lines when dealing with the opioid crisis, taking into consideration alternative pain management options is extremely important. Dentists prescribe $12 \%$ of all immediate-release opioids in the US [13]. Also, for patients aged 10 to 19 , dentists are the main prescribers of opioids [14]. Teenagers and young adults are especially at risk of becoming opioid-dependent after they have been prescribed pain killers from their dentists or oral surgeons. A study from the Stanford School of Medicine, published in Journal of American Medical Association (JAMA) Internal Medicine found that 5.8\% 
out of the 15000 teenagers and young adults included in the study were diagnosed with opioid abuse after the initial prescription was given [15].

Getting the patient into a relaxed state of mind to relieve the anxiety and fear before a dental procedure is in many ways essential for a successful dental visit. Anxiety, fear, and pain are all linked. Patients will endure significant pain for prolonged periods because of their anxiety and anticipation of dental visit. Talking to the patient and making them feel in control are major factors in easing the anxiety before the start of an appointment [16]. Also using the tools that are available for the dentist like nitrous oxide, which is a highly effective tool but underused, will help with anxiety [17]. Digital nitrous is a recently developed method that uses virtual reality (VR) goggles with relaxing scenery while the patient is undergoing the dental procedure [18]. VR is used to reduce pain by distraction. When the mind is deeply immersed in an experience it shuts off external stimuli and thus takes away the feeling of pain, at least temporarily. It was found that VR was able to reduce pain 3 times more than conventional distraction/relaxing methods like TV [19]. The current guidelines set by the Centers for Disease Control (CDC) and the American Dental Association (ADA) for prescribing opioids in the dental setting recommend that clinicians should prescribe non-opioid analgesics as the first line of pain control [20]. When opioids are needed, clinicians should only prescribe the lowest effective dose of immediate-release opioids with the proper quantity to avoid "free-floating" pills. The recommended sufficient dosage is three days or less, keeping in mind that dosages of more than seven days will rarely be needed. Although some patients expect to receive opioid medication after dental procedures, a simple conversation with the patient will likely convince them to try non-opioid alternatives (Figure 3).

Figure 2
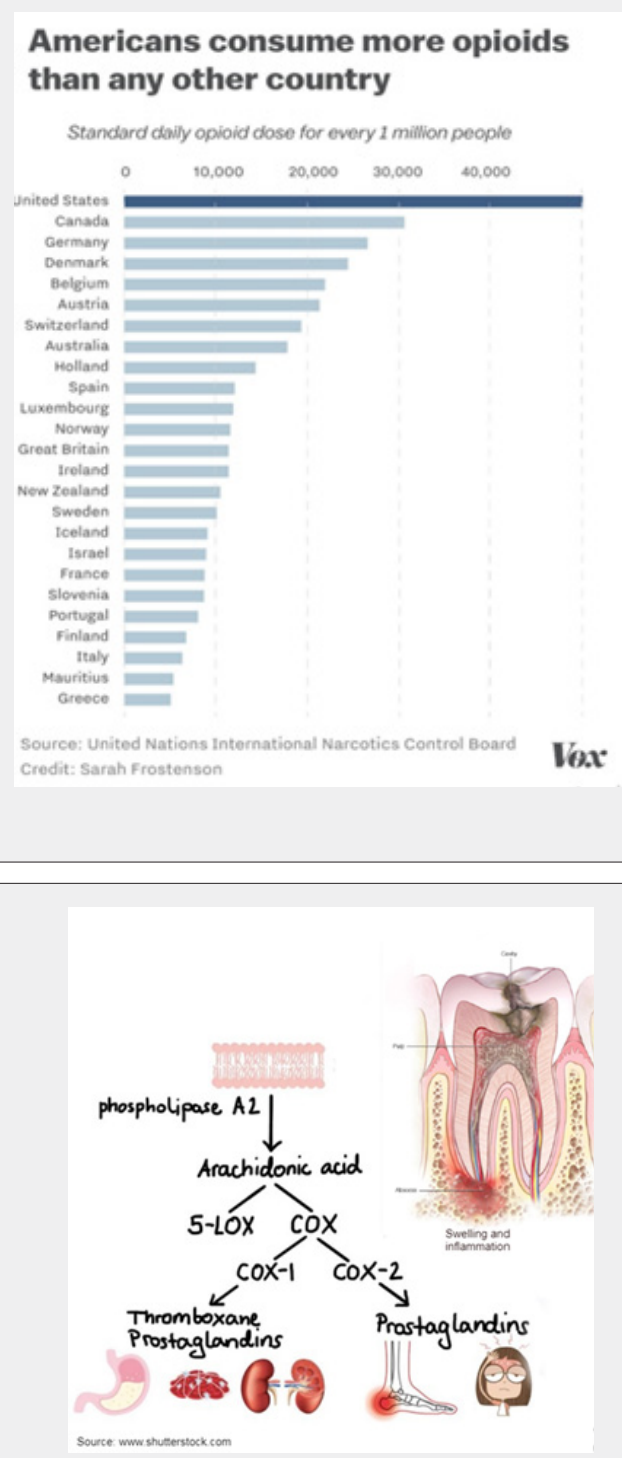

Figure 1: Clinical photograph showing patient's upper lip with the sinus, missing 21 and obliteration of the buccal sulcus in the anterior maxillary region. 
Regardless of the dental procedure, multiple studies show the efficiency of over-the-counter analgesics like Non-Steroidal Anti-inflammatory drugs (NSAIDs) and Acetyl-Para-Aminophenol (APAP). The combination of using both medications gives a significantly underrated analgesic effect that can be opioid sparing [21]. There is evidence indicating NSAIDs being more effective than opioids for severe pain in some situations [22]. The problem with dispensing medication for patients after a surgical dental procedure is that it is based on the assumption that all patients will experience pain that requires opioids. While $95 \%$ or more of patients who go through surgical dental procedures experience moderate to severe pain, not all of them require opioids for pain management yet all of them are given opioid prescriptions. Some can manage their pain by using a combination of OTC medications and others feel the need to use their prescriptions. The practice of prescribing all patients with opioids and then not all patients using their prescriptions results with unused and unsecured pills sitting in irresponsible hands. Like mentioned earlier, the vast majority of misused opioids are obtained through friends and family.
A study conducted in the state of Utah indicated that $72 \%$ of subjects included in the study had leftover opioids after the prescription was filled, and almost all of them kept the leftovers and did not dispose of them [23]. Even though this data is specific to only one state, it shows the importance that providers need to correctly estimate the needed supply of opioids depending on the procedure done. Another alternative for acute post-procedural pain is a long-acting local anesthetic called Exparel. The drug was first introduced to be mainly used in bunionectomy and hemorrhoidectomies [24]. Since then the use of the drug has been vastly expanded to include orthopedic surgery, oral surgery, women's health surgery, and general surgery [25]. Exparel was recently picked up by the dental field and is slowly gaining traction for its effectiveness in eliminating the need for opioid medications after dental procedures. This is possible because Exparel can produce local anesthesia at the site of injection for up to 5 days after the procedure as seen in some clinical trials [26]. The drug is used as a part of a multimodal approach to post-procedural pain management to reduce the need for opioids dispensed after surgery (Figure 4).

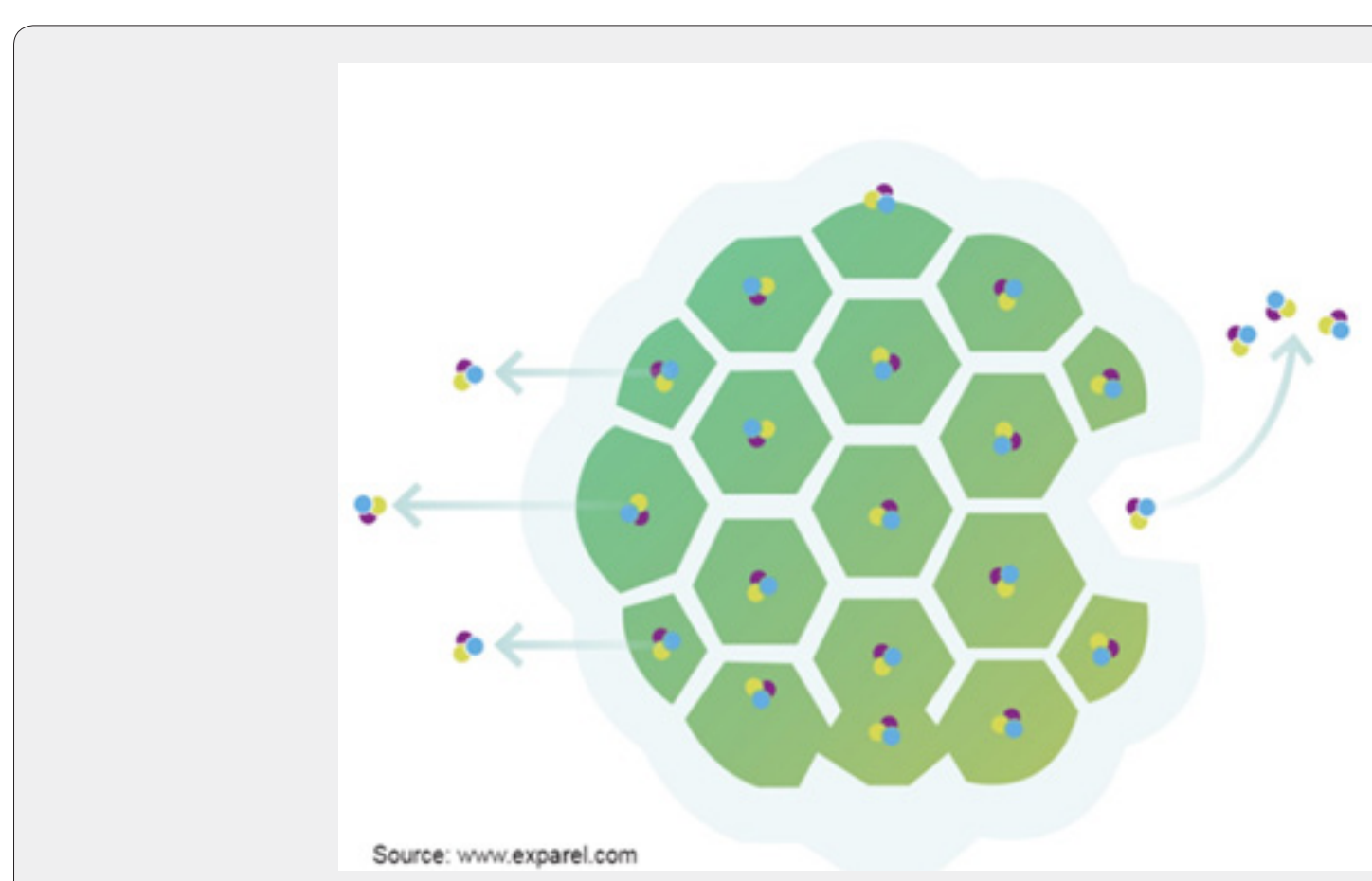

Figure 4

The active pharmaceutical ingredient in Exparel is bupivacaine. Bupivacaine, similar to other local anesthetics used, achieves local anesthesia by binding to the hydrogen ions in the intracellular space and moves into the pores of the voltage-gated sodium channel (VGNaC) thus blocking the passage of sodium through the channel thereby inhibiting nerve conduction [27]. Bupivacaine molecules in Exparel are encapsulated in liposomes that are organized in honeycomb-like structures that prevent the bupivacaine from being metabolized so quickly [28]. This form of drug delivery is known as the DepoFoam drug delivery system. Bupivacaine is slowly released from the lipid in a complex mechanism where the capsules of the lipid rearrange and as a result release the anesthetic over an extended period [28]. While the efficacy of Exparel depends on different factors like the injection technique and the volume required to cover the area, the drug has been wildly successful in minimizing the 
need for opioid medications for post-procedural pain. According to the information posted on the health care provider Exparel Website, more than 6.5 million patients have received the drug since 2012 , and there has been a $78 \%$ reduction in the overall consumption of opioids when Exparel is used [28]. One of the challenges dental providers are facing with Exparel is the cost coverage. There is currently only one insurance company, Aetna, that offers reimbursement of cost when Exparel is used with third molar extractions. Although this can vary depending on the patient's specific coverage plan. Also, Exparel only comes in two vial sizes $10 \mathrm{cc}$ or $20 \mathrm{cc}$, where only 2-3cc might be needed per site. If there was more vial size options that correspond to the exact amount needed it could make it more affordable for the patient. An even better option is to lobby more insurance companies to agree to cover the cost of the drug given the benefit it provides. In a patient survey with a single oral and maxillofacial surgeon in Cleveland Ohio where over 100 patients were provided Exparel for pain control on various sort of dentoalveolar procedures the opioid utilization was dropped by over $90 \%$. Patient satisfaction with Exparel was over $99 \%$ as one patient raised concern that it took over 8 hours from time of injection to provide relief.

\section{Conclusion}

In conclusion, as health care providers we are responsible for the health and well-being of the patients that we treat. Given that there is an increased risk that some of our patients may become physically dependent on opioids, it is our responsibility to create a conscious effort in seeking alternative post-procedural pain management options. This means that dental providers must always stay up to date with practicing guidelines issued by governmental bodies. As well as setting an example to the rest of the health care field in using result-yielding alternative pain management options. And most importantly not falling into the trap of complacency during everyday practice. As Paulo Coelho said, "If you think adventure is dangerous, try routine; it is lethal".

\section{References}

1. Paulina Świeboda, Rafał Filip, Andrzej Prystupa, Mariola Drozd (2013) Assessment of pain: types, mechanism and treatment. Ann Agric Environ Med 20(Special Issue 1): 2-7.

2. https://ultimatecieguide.files.wordpress.com/2017/03/sarafinohealth-psychology-biopsychosocial-interactions-edition-7.pdf

3. https://www.fauchard.org/publications/39-man-and-pain-eternalpartners

4. https://www.sciencehistory.org/historical-profile/humphry-davy

5. https://www.worldofmolecules.com/drugs/novocaine.htm

6. https://www.nytimes.com/2019/08/26/health/oklahoma-opioidsjohnson-and-johnson.html

7. http://documents.latimes.com/oxycontin-press-release-1996/
8. Rachel N Lipari, Arthur Hughes (2017) How People Obtain the Prescription Pain Relievers they Misuse. Center for Behavioral Health Statistics and Quality.

9. https://www.drugabuse.gov/publications/research-reports/misuseprescription-drugs/what-scope-prescription-drug-misuse

10. Katie J Suda, Michael J Durkin, Gregory S (2019) Calip Comparison of Opioid Prescribing by Dentists in the United States and England. Jama network 2(5): e194303.

11. https://www.aetnadental.com/professionals/dental-overall-health/ opioid-alternatives.html

12. Richie Onwuchekwa Uba, Kofi Ankoma Darko, Sharon K Park (2020) International comparison of mitigation strategies for addressing opioid misuse: A systematic review. Journal of the American Pharmacists Association 60(1): 195-204.

13. Nora D Volkow, Thomas A McLellan, Jessica H Cotto (2011) Characteristics of Opioid Prescriptions in 2009. JAMA 305(13): 12991301.

14. Alan R Schroeder, Melody Dehghan, Thomas B Newman (2019) Association of Opioid Prescriptions from Dental Clinicians for US Adolescents and Young Adults with Subsequent Opioid Use and Abuse. JAMA Intern Med 179(2): 145-152.

15. Deva Priya Appukuttan (2016) Strategies to manage patients with dental anxiety and dental phobia: literature review. Clin Cosmet Investig Dent 8: 35-50.

16. Mark Griffiths (2014) Hypnosis for dental anxiety. Dent Update 41(1): 78-80.

17. Karin Tanja-Dijkstra, Sabine Pahl, Mathew P White, Melissa Auvray, Robert J Stone, et al. (2017) The Soothing Sea: A Virtual Coastal Walk Can Reduce Experienced and Recollected Pain. Sage 50(6): 599-625.

18. Brennan Spiegel, Garth Fuller, Mayra Lopez, Taylor Dupuy, Benjamin Noah, et al. (2019) Virtual reality for management of pain in hospitalized patients: A randomized comparative effectiveness trial. PLOS ONE 14(8): e0219115.

19. https://www.dentallearning.org/course/LocalAnesthetic/Local\%20 Anesthetic.pdf

20.https://www.cdc.gov/acute-pain/dental-pain/index. html\#: :text=Follow $\% 20$ the $\% 20$ CDC $\% 2$ gguidelines $\% 3 \mathrm{~A} \% 20$ 'Clinicians,days\%20will\%20rarely\%20be\%20needed.

21. Paul A Moore, Elliot V Hersh (2013) Combining ibuprofen and acetaminophen for acute pain management after third-molar extractions: translating clinical research to dental practice. J Am Dent Assoc 144(8): 898-908.

22. https://www.cdc.gov/mmwr/preview/mmwrhtml/mm5906a1.htm

23. https://www.centerwatch.com/directories/1067-fda-approveddrugs/listing/3505-exparel-bupivacaine-liposome-injectablesuspension

24. https://www.exparel.com/hcp/exparel-vs-pumps-and-catheters

25. https://www.exparel.com/patient/faq

26. Daniel E Becker, Kenneth L Reed (2012) Local Anesthetics: Review of Pharmacological Considerations. Anesth Prog 59(2): 90-101.

27. https://www.fda.gov/media/111001/download

28. https://www.exparel.com/hcp/index 
(C) This work is licensed under Creative (1) Commons Attribution 4.0 License DOI: 10.19080/ADOH.2020.13.555864
Your next submission with Juniper Publishers will reach you the below assets

- Quality Editorial service

- Swift Peer Review

- Reprints availability

- E-prints Service

- Manuscript Podcast for convenient understanding

- Global attainment for your research

- Manuscript accessibility in different formats ( Pdf, E-pub, Full Text, Audio)

- Unceasing customer service

Track the below URL for one-step submission https://juniperpublishers.com/online-submission.php 